HELMINTHOLOGIA, 54, 4: 298 - 306, 2017

\title{
Epidemiology of Opisthorchis viverrini in an endemic area of Thailand, an integrative approach
}

\section{S. PRAKOBWONG ${ }^{1,2}$, W. GUNNULA ${ }^{3}$, S. CHAIPIBOOL ${ }^{4}$, B. NIMALA ${ }^{4}$, J. SANGTHOPO ${ }^{4}$, N. SIRIVETTHUMRONG ${ }^{2,5}$, A. RIBAS ${ }^{1 *}$}

'Department of Biology, Udon Thani Rajabhat University, Udon Thani, 41000, Thailand, E-mail: aribas@ub.edu; 'Liver Fluke Research and Academic Outreach Unit, Faculty of Science, Udon Thani Rajabhat University, Udon Thani, 41000, Thailand; ${ }^{3}$ Department of

Biology, Faculty of Science, Nakhonphanom University, Nakhon Phanom, 48000, Thailand; ${ }^{4} \mathrm{Nam}-P$ Phon Hospital of Health Promotion, Nong Wua Sor district, Udon Thani, 41000, Thailand; ${ }^{5}$ Udonthani Provincial Health Office, Ministry of Public Health, Thailand

Article info

Received April 11, 2017

Accepted July 6, 2017

\begin{abstract}
Summary
An integrated epidemiological study of Opisthorchis viverrini consisting of risk factors analysis and parasitation prevalence determination in humans, as well as the assessment the roles of host reservoirs (dogs and cats) and intermediate hosts (cyprinid fish) was carried out in the vicinity of Huay Luang dam, Udon Thani province, in the north east of Thailand. The survey was conducted from June 2014 to July 2016 in three sub-districts. Fecal samples were collected from 5,347 participants in 22 villages and the overall prevalence of 0 . viverrini was found to be $31.5 \%$. Risk factors for liver fluke infection were determined from questionnaires analysis which sought demographic data and information about the eating behaviors of the participants. The behavior of raw fish consumption correlated significantly with parasitation in the studied areas. The risk factors for $\mathrm{O}$. viverrini infection in humans were associated significantly with age, education, and habitation within a two kilometer range from nearby water and food sources. Questionnaires showed that the drug treatments were not always appropriate. The stools of domestic dogs $(n=468)$ and cats $(n=262)$ collected for parasitological analysis and results showed that the zoonotic role of dogs should not be underestimated as has been done previously/in past. The incidence of $O$. viverrini metacercariae in four cyprinid fishes Barbonymus gonionotus $(n=124)$, Cyclocheilichthys repasson $(n=843)$, Hampala dispar $(n=276)$ and Henicorhynchus siamensis $n=946$ ), were determined and overall values ranged from $2.4 \%$ to $23.1 \%$. There was a seasonal variation in metacercariae intensity which ranged from $1-125$ metacercariae per fish. The study concluded that transmission ecology should be taken into consideration in developing of control strategies against opisthorchiasis.
\end{abstract}

Keywords: cholangiocarcinoma; Opisthorchis viverrini; epidemiology; reservoirs; intermediate host

\section{Introduction}

Chronic infection caused by the Southeast Asian liver fluke (Opisthorchis viverrini) is a critical risk factor for the development of the bile duct cancer cholangiocarcinoma (CCA), what is a major health concern in South-East Asia (Thailand, Laos People's Democratic Republic, Cambodia and central Vietnam) (Sripa et al., 2010; Aunpromma et al., 2012; Miyamoto et al., 2014; Kaewpitoon et al.,
2015; Khuntikeo et al., 2016). In Thailand at least 6 million people are estimated to be currently infected (Andrews et al., 2008). Opisthorchis viverrini infection in humans and in dog and cat host reservoirs (Sithithaworn \& Haswell-Elkins, 2003) occurs via the consumption of a raw or uncooked fish which contains infective metacercariae (Sripa et al., 2007; Donthaisong et al., 2014; Madsen et al., 2015; Onsurathum et al., 2016) In addition, fermented fish also contain high concentrations of nitrosamines that are

\footnotetext{
* - corresponding author
} 
known to be potent human carcinogens (Thamavit et al., 1993). Once metacercariae are ingested they migrate to the intrahepatic bile duct via the common bile duct and once the fluke becomes gravid (in about 30 days) the eggs are excreted by feces (Harinasuta \& Harinasuta, 1984). Species diversity and the abundance of cyprinid fish, which are available all year round, are involved in the life cycle across north east Thailand and facilitate the maintenance of life cycle of this trematode. A study across 20 provinces in the north east of Thailand showed that 13 of them are tested positive for the occurrence to this parasite (Pinlaor et al., 2013).

Despite detected overall decline in the prevalence of this helminthiasis in Thailand (from $14.7 \%$ to $8.7 \%, 1980$ - 1981, Preuksaraj et al., 1982) the last national survey performed in 2009 (Sithithaworn et al., 2012), in the provinces of the north east of Thailand, detected the prevalence still as high as $50 \%$ in numerous areas (Sithithaworn et al., 2012). Several reasons can at least partially explain the difficulties associated with reduction of opistorchiasis prevalence. Primarily, it is well rooted tradition of uncooked or undercooked fish (Cyprinidae) consumption (Prasongwatana et al, 2013). Another strongly held belief in the north east Thailand culture that contributes to the maintenance of high prevalence of this trematode is that drinking of strong alcohol can kill the worms (Grundy-Warr et al., 2012).
To our knowledge, an integrated study consisting of parasite prevalence and risk factors analysis in humans as well as the assessment the role of intermediate (cyprinid fish) and host reservoirs (dogs and cats) has never been carried out. Despite antiparasitic treatments being frequently used, which in principle can be highly effective, the prevalence and reinfection rates for this liver fluke remains still high (Saengsawang et al., 2016). To improve this situation a better understanding of the epidemiology of this foodborne parasite and the human factors relevant to its treatment are required to examine.

The present study aims to fill this gap by determining the distribution (prevalence and intensity) across several vertebrates that are essential to the life cycle of this trematode. We have examined areas which are endemic (the north east of Thailand) and analysed the risks of the population inhabiting this territories.

\section{Materials and Methods}

\section{Study area and fecal sample collection}

The survey was conducted in the vicinity of Huay Luang dam, the largest reservoir in the upper part of the north east of Thailand between June 2014 and July 2016. Three sub-districts (Nam Phon, Nong Bua Ban and Khok Sa-ard) of Udon Thani province, where
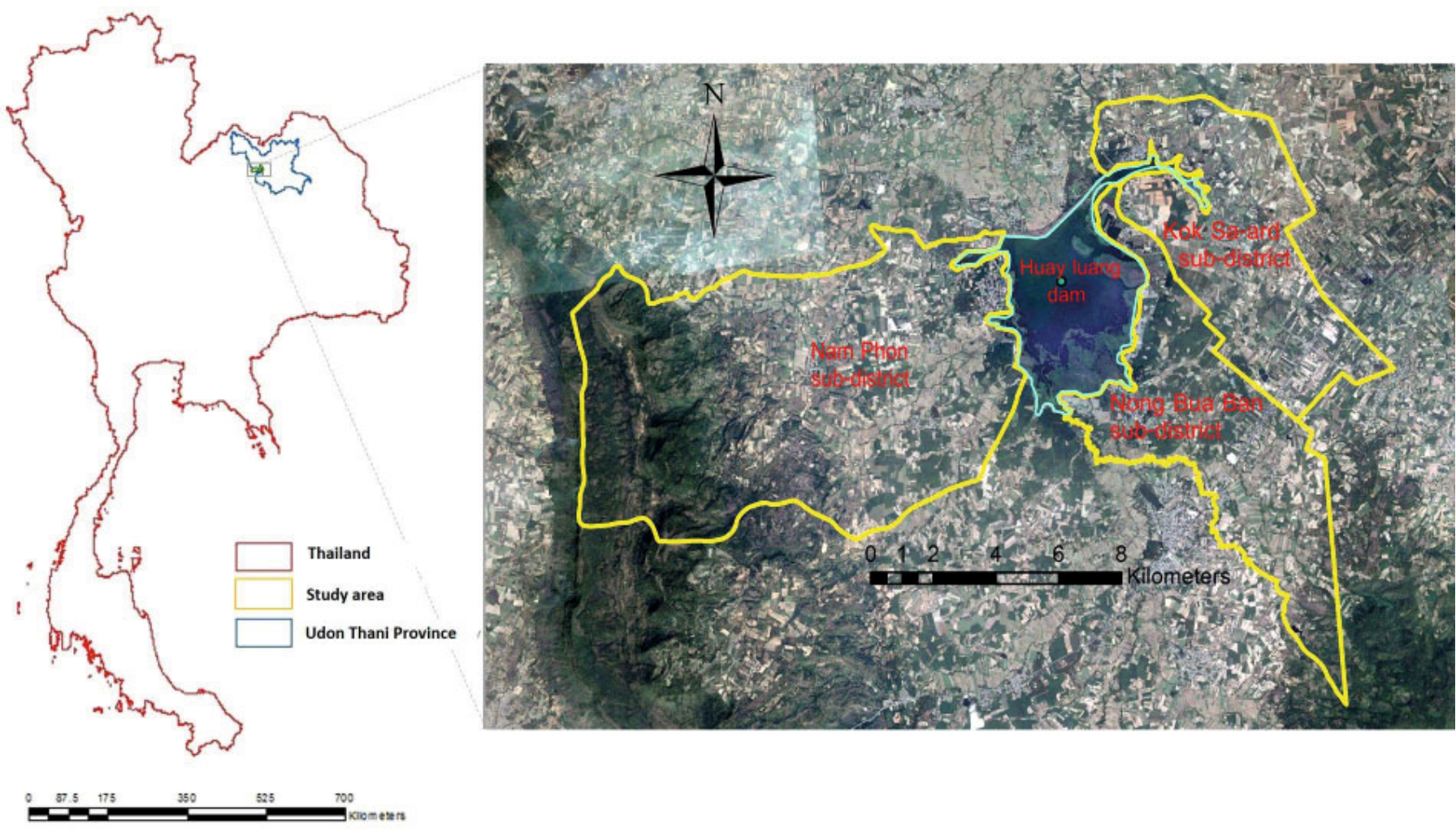

Fig. 1. Map of the surveyed area. 
at least 30,000 people reside in an area of $247 \mathrm{~km}^{2}$, were included in this study (Fig. 1). Fecal samples from 22 villages were collected from 5,347 participants and fixed in $10 \%$ formalin before being transported to a laboratory at the Udon Thani Rajabhat University. Official letters explaining the aim of the program, the development of strategies for opisthorchiasis control were sent to the heads of villages, community leaders, school principals, the children's parents and to the individual households. Visits were made to each village to explain the benefits of the survey as well as the National Opisthorchiasis Control Programme. The study protocol was approved by the Human Ethics Committee of the Ministry of Public Health.

Risk factors for the liver fluke infection were evaluated by using questionnaires to collect demographic data such as age, sex, occupation (agriculture or public servant), monthly income (less than or greater than 10,000 bahts) and smoking habit (yes/no) together with the information about previous parasitic treatments and eating behaviours.

The stools of domestic dogs and cats were collected by confining them to the house of their owners. This particular approach allows performing the correlation between fecal sample results with the data obtained from the human occupants living in the same house. The samples were fixed in $10 \%$ formalin and then transported to a laboratory at Udon Thani Rajabhat University for parasitological analysis. The Animal Ethics Committee of the Udonthani Rajabhat University approved this part of the study.
Modified formalin ethyl-acetate concentration technique

The quantitative formalin ethyl acetate concentration technique with slight modification was performed. One gram of feces was fixed with $10 \%$ formalin and filtered through 2 layers of gauze after which the suspension was centrifuged at 2,500 rpm for 3 minutes (EBA20S, Hettich, Singapore). The pellet was then re-suspended in $10 \mathrm{ml}$ of $10 \%$ formalin and $3 \mathrm{ml}$ of ethyl-acetate and centrifuged at 2,500 rpm for a another 3 minutes. Lipid floating on the supernatant was removed after separating it by ringing with an applicator stick from the walls of the tube. One $\mathrm{ml}$ of $10 \%$ formalin was then added to the sediment and the number of eggs per gram of liver fluke infected feces was assessed individually by two experienced researchers.

\section{Isolation of metacercariae from cyprinid fishes}

Cyprinid fish traditionally caught by local people living around the Huay Luang dam were examined for the metacercariae within 24 hours after the collections. The sampling was conducted on monthly basis from February 2014 to January 2016 and the isolation of 0 . viverrini metacercariae was achieved by pepsin digestion (Pinalor et al., 2013). Each whole fish was identified at the species level, weighed individually and minced by electric blender before being immersed in an aqueous solution containing $0.25 \%$ pepsin, $1.5 \% \mathrm{HCl}$ and $0.85 \% \mathrm{NaCl}$. The mixture was then incubated at $37^{\circ} \mathrm{C}$ for 2 hours before the digested fish were filtered using 4 consecutive metal sieves (1100; 350; 250 and $140 \mu \mathrm{m}$

Table 1. Characteristics and prevalence of $O$. viverrini in humans.

\begin{tabular}{lcccc}
\hline Characteristics & $\begin{array}{c}\text { Stools } \\
(\mathbf{n})\end{array}$ & $\begin{array}{c}\text { Positive } \\
(\mathbf{n})\end{array}$ & $\begin{array}{c}\text { Prevalence } \\
(\%)\end{array}$ & $\begin{array}{c}\text { Average of EPG } \\
(\text { range })\end{array}$ \\
\hline Gender & & & & \\
Male & 2,565 & 742 & 28.9 & $305.1(12-3,594)^{*}$ \\
Female & 2,782 & 946 & 34.0 & $43.2(7-160)$ \\
\hline Age & & & & \\
$<25$ & 825 & 61 & 7.4 & $33.6(7-176)$ \\
$25-35$ & 1,074 & 385 & 35.8 & $63.1(36-296)$ \\
$36-45$ & 1755 & 512 & 29.1 & $113.7(33-3,594)$ \\
$46-55$ & 1,149 & 531 & 46.2 & $231.8(24-1,686)^{*}$ \\
$>55$ & 544 & 199 & 36.5 & $23.5(11-464)$ \\
\hline Sub-district & & & & $162.8(7-3,594)^{*}$ \\
Nam Phon & 2,762 & 1,374 & 49.7 & $145.3(16-254)$ \\
Nong Bua Ban & 1,518 & 221 & 14.5 & $145.3(27-653)$ \\
Khok Sa-ard & 1,067 & 93 & 8.7 & $74.2(7-3,594)$ \\
\hline Total & 5,347 & 1,688 & 31.5 & \\
\hline${ }^{*}-$ significance $p<0.05$ & & & &
\end{tabular}


Table 2. Prevalence and intensity of $O$. viverrini in dog, cat and human hosts.

\begin{tabular}{lcccc}
\hline Host & $\begin{array}{c}\text { Stools } \\
(\mathbf{n})\end{array}$ & $\begin{array}{c}\text { Positive } \\
(\mathbf{n})\end{array}$ & $\begin{array}{c}\text { Prevalence } \\
(\%)\end{array}$ & $\begin{array}{c}\text { Average EPG } \\
\text { (range in each village) }\end{array}$ \\
\hline Dogs & 468 & 81 & 17.3 & $78.1(21-169)$ \\
Cats & 262 & 64 & $24.4^{*}$ & $145.3(36-296)^{*}$ \\
Humans & 5,347 & 1,688 & $31.5^{* *}$ & $74.2(7-3,594)^{*}$ \\
Total/Average & 6,077 & 1,833 & 30.1 & $82.1(7-3,594)$ \\
\hline${ }^{*}$ - significant $p<0.05 ;{ }^{*}$ - significant higher than $p<0.05$ &
\end{tabular}

apertures). Filtered fish pellets were sedimented in $0.85 \% \mathrm{NaCl}$ in a sediment jar several times until the supernatant was clear (the details of the technique are given in (Pinlaor et al., 2013)). Metacercariae of $O$. viverrini in the sediment were identified and counted using a stereo-microscope (Motic® DMW-143, USA).

\section{Statistical analyses}

Data are presented as average infection percentages. One-way ANOVA was used to compare the infection intensity (eggs per gram - EPG) in feces and compared with infective rates of metacercariae between different species of fish. Student's t-test and chi-squared tests were used for parametric and non-parametric analysis, respectively. Pearson's correlation coefficient was used to analyse correlations between parametric data and regression analysis was performed if the data exhibited significant correlations. Odds ratios $(\mathrm{OR})$ were calculated to determine the association between cross sectional data to predict possible risk factors and analysed for all other predictive variables on the basis of logistic regression analysis. Statistical analyses were performed using the SPSS version 11.5 and $p$ values less than 0.05 were considered statistically significant.

\section{Post-treatment}

Humans as well as the host reservoirs (dogs and cats) which were found to be parasitized with 0 . viverrini were cured with praziquantel. This treatment was part of the active prevention and control program implementation performed by the Faculty of Science at the Udonthani Rajabhat University.

\section{Results}

Prevalence and intensity of $\mathrm{O}$. viverrini in human and host reservoirs

A total of 5,347 participants (2,565 males and 2,782 females) representing $40 \%$ of the population older than 20 years old were screened (range: $20-82$ years old, see Table 1). The overall prevalence of 0 . viverrini was $31.5 \%$ (1,688 infected). However, the egg counts were statistically significant between sexes (305.1 EPG in males versus 43.2 EPG in females). As can be seen in Table 1, an age-dependence was found for Opisthorchis infec- tion where infection peaked in the $46-55$ years age range. The highest prevalence (49.7\%) and intensity (162.8, range $7-3,594$ EPG) was found in the Nam Phon sub-district within the Nong Wua Sor district $(p<0.05)$.

The prevalence of 0 . viverrini infection in domestic dogs $(n=$ $468)$ and cats $(n=262)$ is presented in Table 2 . The rates of liver fluke infection in cats $(24.4 \%)$ were significantly higher than in dogs $(17.3 \%)$, while the highest prevalence was found in humans $(31.5 \% ; p<0.05)$. The average EPG in feces collected from dogs was 78.1 (range; 21 - 169) meanwhile the cats EPG was 145.3 (range; $36-296$ ), and in humans the average EPG was 74.2 (range; $7-3,594$ ). Data, confirmed significantly different infection rates in humans and cats when compared to dogs $(p<0.05)$.

\section{Risk factors for 0 . viverrini infection}

A logistic regression analysis and the odds ratios were performed and calculated from 1,634 questionnaires where $31.5 \%$ of participants provided also fecal samples for screening (Table 3 ). The habit of eating a raw fish ( $p=0.04$; OR $=3.1$ ) was found to be important and statistically significant in the areas studied. The univariate model for risk factors in 0 . viverrini human infection were significantly linked with gender $(p=0.067 ; \mathrm{OR}=1.6)$; age $(p \leq$ 0.035 ; $\mathrm{OR}=2.7)$; education $(p \leq 0.000 ; \mathrm{OR}=12.6)$, the residence within a 2 kilometers range of nearby water $(p \leq 0.000 ; O R=2.2)$ and food selection factors $(p=0.041 ; \mathrm{OR}=2.0$ ) were found important. Relating to the drug treatments, most of infected participants lack the knowledge of praziquantel use $(p \leq 0.000 ; \mathrm{OR}=7.4)$ and had no history of parasitic treatment $(p \leq 0.037 ; \mathrm{OR}=3.3)$. Despite the question not being included in the questionnaires, most of the interviewed participants were aware of albendazole treatment. No statistically significant results were found within the occupation ( $p$ $=0.16, \mathrm{OR}=1.1)$, smoking habit $(p=0.51, \mathrm{OR}=1.0)$ or monthly income $(p=0.67, \mathrm{OR}=1.2)$ categories.

Prevalence and intensity of 0 . viverrini metacercaria in cyprinid fishes

Four cyprinid species, that are very common in catchments in the areas studied, were surveyed for 0 . viverrini metacercariae: Barbonymus gonionotus (Pla Ta-Pein in Thai); Cyclocheilichthys repasson (Pla Sai Ton); Hampala dispar (Pla Kasub Jud) and 
Table 3. Analysis of risk factors of 0 . viverrini infection in endemic areas.

\begin{tabular}{|c|c|c|c|c|}
\hline Significant factors & $\begin{array}{c}\text { questionnaires } \\
(\mathrm{n}) \\
\text { (Total } 1,634)\end{array}$ & $\begin{array}{c}\text { Infected } \\
\text { n (\%) }\end{array}$ & $p$ value & $\begin{array}{l}\text { Odd ratio } \\
(95 \% \mathrm{Cl})\end{array}$ \\
\hline \multicolumn{5}{|l|}{ Gender } \\
\hline Male & 784 & $91(11.6)$ & 0.067 & 1.0 \\
\hline Female & 850 & $123(14.4)$ & & $1.6(0.6-12.5)$ \\
\hline \multicolumn{5}{|l|}{ Age } \\
\hline$>50$ years & 532 & $51(9.5)$ & 0.035 & 1.0 \\
\hline$<50$ years & 1,102 & $163(14.8)$ & & $2.7(0.5-6.3)$ \\
\hline \multicolumn{5}{|l|}{ Education } \\
\hline $2^{\text {nd }}$ school and under & 1,328 & $95(7.1)$ & 0.000 & 1.0 \\
\hline Higher than $2^{\text {nd }}$ school & 306 & $119(38.8)$ & & $12.6(3.2-18.0)$ \\
\hline \multicolumn{5}{|l|}{$2 \mathrm{~km}$ close to water } \\
\hline No & 695 & $71(10.2)$ & 0.000 & 1.0 \\
\hline Yes & 939 & $143(15.2)$ & & $2.2(0.5-6.6)$ \\
\hline \multicolumn{5}{|c|}{ Life style and food selective vision } \\
\hline by value of nutrition & 612 & $47(7.6)$ & 0.041 & 1.0 \\
\hline by tradition & 1,022 & $167(16.3)$ & & $2.0(0.7-8.1)$ \\
\hline \multicolumn{5}{|c|}{ Knowledge of praziquantel treatment } \\
\hline Yes & 769 & $51(6.6)$ & 0.000 & 1.0 \\
\hline No & 865 & $163(18.8)$ & & $7.4(1.2-14.3)$ \\
\hline \multicolumn{5}{|c|}{ History of parasitic treatment } \\
\hline Ever & 738 & $61(8.2)$ & 0.037 & 1.0 \\
\hline Never & 896 & $153(17.0)$ & & $3.3(0.6-13.6)$ \\
\hline \multicolumn{5}{|l|}{ Eat raw fish in last 1 year } \\
\hline Never & 1,044 & $115(11.0)$ & 0.044 & 1.0 \\
\hline Often & 590 & $99(16.7)$ & & $3.1(0.9-11.2)$ \\
\hline
\end{tabular}

Henicorhynchus siamensis (Pla Kao Na), (see details provided in Table 4). The highest infection prevalence among the four species of fish was found in $\mathrm{H}$. siamensis $(23.1 \%, \mathrm{n}=946)$ and then with decreasing order in $C$. repasson $(15.8 \%, n=843), H$. dispar $(5.4 \%, n=276)$ and $B$. gonionotus $(2.4 \%, n=124)$. The infection intensity in each fish ranged from $1-125$ metacercariae. Significantly higher numbers were found in $\mathrm{H}$. siamensis and $\mathrm{C}$. repasson when compared with $H$. dispar and $B$. gonionotus $(p<0.05)$. Figure 2 shows that the seasonal average intensity of metacercariae infection during the January to April period was significantly higher in $H$. siamensis and C. repasson (infection range: $25 \%$ $33 \%$; intensity: $13-125$ metacercariae/fish, $p<0.05$ ), whereas decreased levels of infection were found during July to October period (range: $1 \%-8 \%$; intensity: $1-18$ metacercariae/fish, $p<0.05$ ).

\section{Discussion}

Our study suggests a well-established life cycle for 0 . viverrini in the Huay Luang dam area as it was simultaneously detected by high prevalence levels in humans, dogs, cats and cyprinid fish, each associated with considerable parasite loads.

In humans, a National survey of helminthiasis in Thailand in 2009 (Sithithaworn et al., 2012) showed that the north east of Thailand is an area in which 0 . viverrini is known to be endemic, where the prevalence across this region vary from $4.6 \%$ to $60.8 \%$. The values reported in our recent study are higher $(31.5 \%)$ when compared with the average values found in Udon Thani Province (13.8 \%) (Sithithaworn et al., 2012). In this study, at least 1,688 participants were infected suggesting the existence of a high risk 


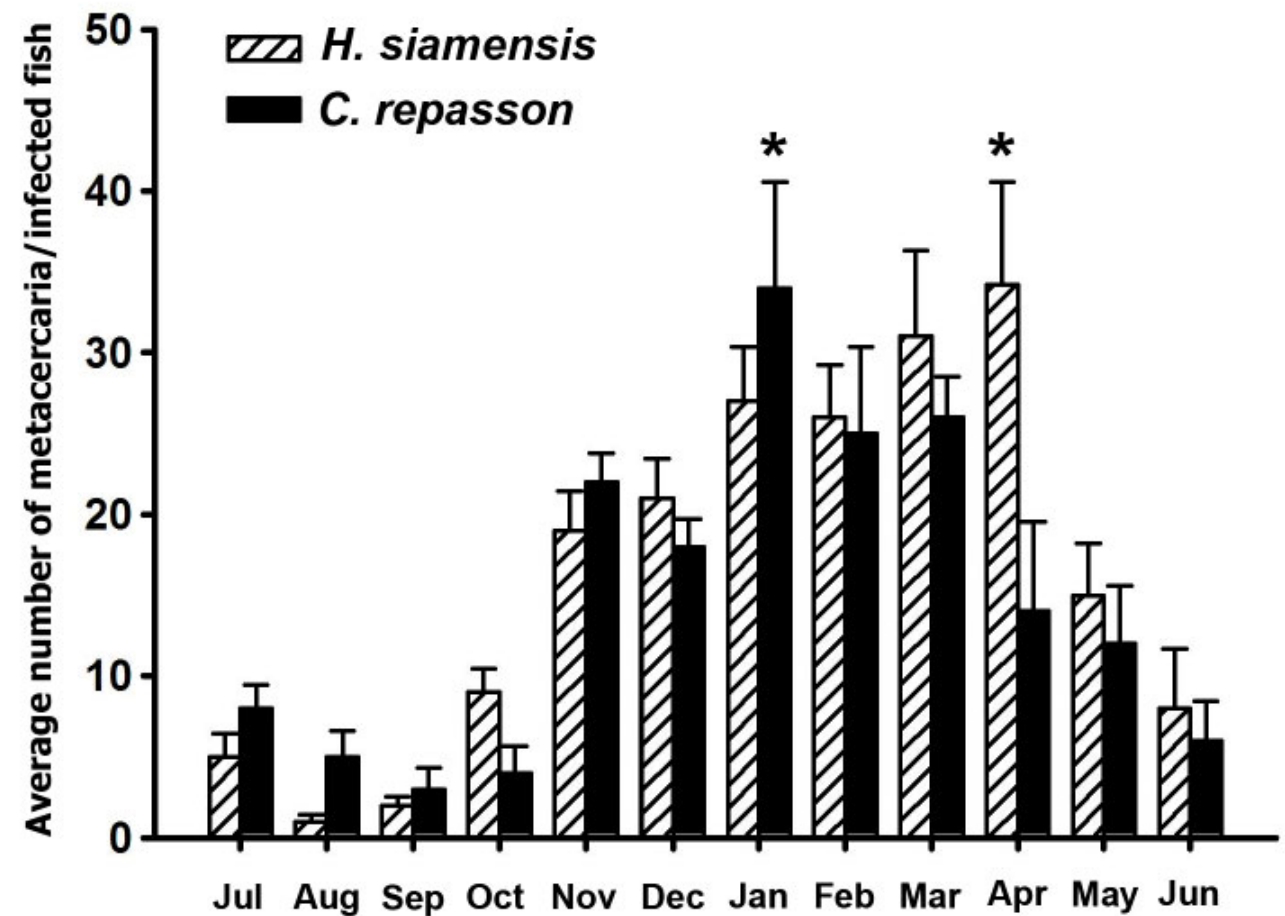

Fig. 2. Seasonal distribution of metacercariae in two fish species.

$$
\left({ }^{*}-\text { significant } p<0.05\right)
$$

area for the development of cholangiocarcinoma.

We proved that the risk factors for 0 . viverrini infection were significantly related with habits of eating raw or undercooked infected-fish (Sripa et al., 2007) an activity that is common in all areas in which opisthorchiasis is spread (Grundy-Warr et al., 2012). This habits suggest that the selection of food in this area is based on tradition and not on nutritional or other health values.

The higher parasite numbers in males than females (305.1 EPG in males versus 43.2 EPG in females) can be accounted for the significant differences in food habits between genders, where male consumption of a raw fish (koi pla) with alcohol drinking is associated with masculine behaviour (Grundy-Warr et al., 2012).
This probably also explains the association with age found in the present study ( $p \leq 0.035$; OR $=2.7$ ), as compounded by the cumulative effects of the adult trematode living for over 10 years (Harinasuta \& Harinasuta, 1984). The higher parasite quantities have been exacerbated by the observation that a considerable fraction of the surveyed population has never been treated with antihelminthics. As previously demonstrated all these risk factors can also be linked with the education level (Chaiputcha et al., 2015). Our survey indicates that a lesser education, up to the level of primary school or lower, was associated with higher risk of infection. In contrast, the education beyond secondary school level or above was a factor with significant protective outcome.

Table 4. Prevalence and intensity of 0 . viverrini metacercariae in cyprinid fishes.

\begin{tabular}{lccccc}
\hline Host & Number of fish & $\begin{array}{c}\text { Number testing } \\
\text { positive }\end{array}$ & $\begin{array}{c}\text { Prevalence } \\
(\%)\end{array}$ & $\begin{array}{c}\text { Average } \\
\text { metacercaria/gram } \\
\text { (range) }\end{array}$ & $\begin{array}{c}\text { Average } \\
\text { metacercaria/fish } \\
\text { (range) }\end{array}$ \\
\hline Henicorhynchus siamensis & 946 & 219 & 23.1 & $3.1(2-24)^{*}$ & $25.9(1-125)^{*}$ \\
Cyclocheilichthys repasson & 843 & 134 & 15.8 & $1.9(1-17)^{*}$ & $15.4(2-33)^{*}$ \\
Hampala dispar & 276 & 15 & 5.4 & $0.3(1-12)$ & $2.4(1-15)$ \\
Barbonymus gonionotus & 124 & 3 & 2.4 & $\mathrm{ND}$ & $\mathrm{ND}$ \\
Total/Average & 2,189 & 371 & 16.9 & $1.7(1-24)$ & $14.3(1-125)$ \\
\hline
\end{tabular}

* - significant $p<0.05 ., \mathrm{ND}=$ Not determined 

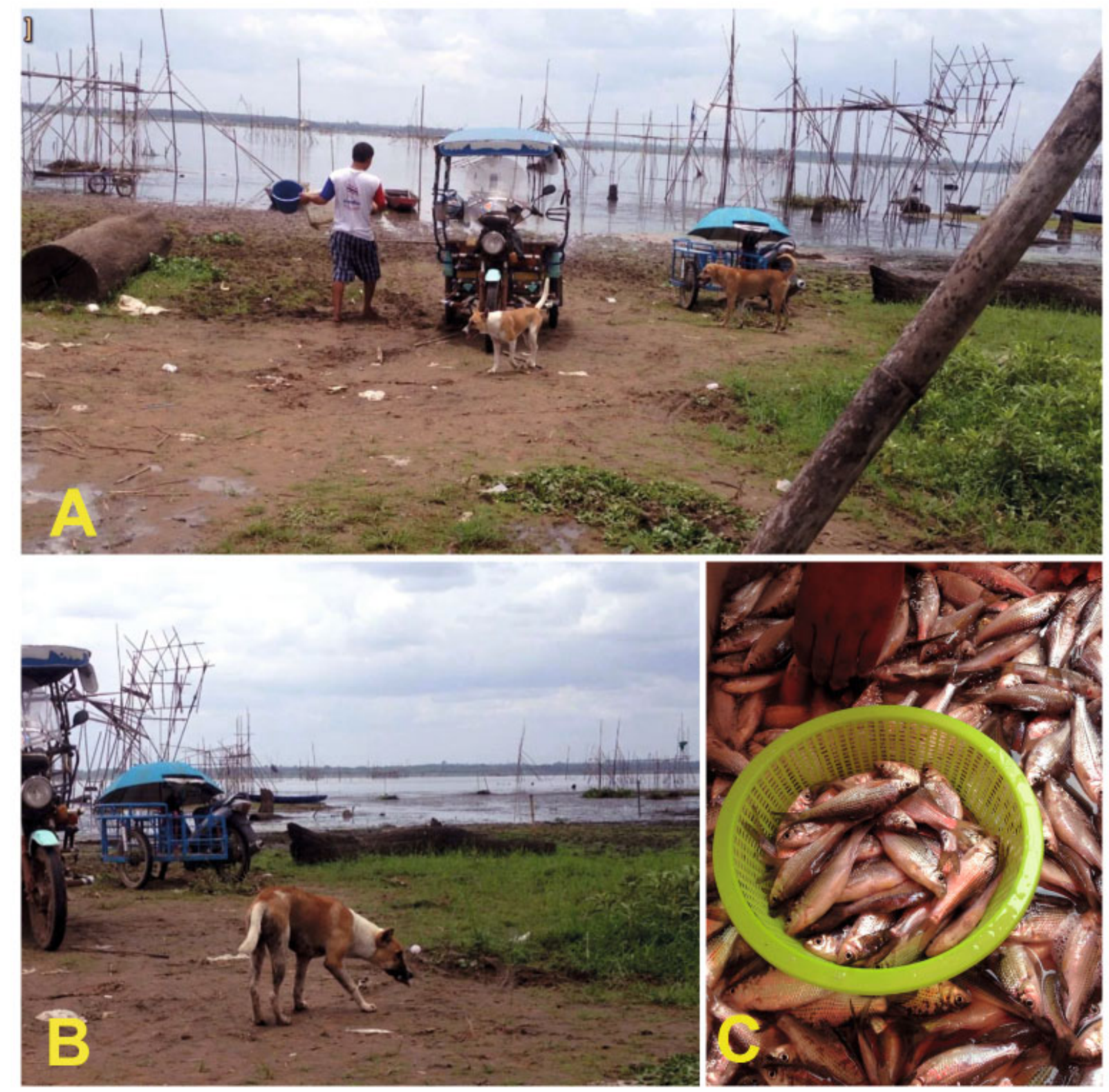

Fig. 3. Dogs eating fish in Huay Luay dam. A; dogs waiting for discarded fish from the fishermen's, B ; dog eating decay fish, C; collected fish for metacercaria detection.

Related to the environment, the population living within two kilometers of water had a closer relationship with fishing activities and consequently a higher probability of consuming parasitized fish. Additionally, the transmission from human/dog/cat feces to Bithynia snails inhabiting the dam should be more likely. Therefore the elimination of fecal contamination from water is imperative to interrupt the transmission of disease.

Most of the participants did not know about the praziquantel effectivity and significant success in the history of disease treatment. Despite the question not being included in the questionnaire most of the surveyed population was aware of the antihelmintic drug albendazole. The praziquantel is the only drug that has been shown to be effective against $O$. viverrini infection in the eastern Thailand where a single dose (40 mg/kg) shows $98 \%$ efficiency rate (Pungpark, Bunnag, \& Harinasuta, 1984). Albendazole is not approved for the treatment of 0 . viverrini infection by the U.S. Food and Drug Administration (CDC, 2012). These may be related to the cost of treatment. Praziquantel treatment is relatively expensive (80 bahts) in comparison to albendazole (30 bahts). A better communication between the Health Care Units and the affected communities would enhance the antihelminthic treatment and will help avoid of an inappropriate self-treatment.

As previously reported by Sithithaworn et al. (1997) our results indicate on a seasonal variation in the prevalence of metacercariae 
(Fig. 2). Despite these differences during the year, the metacercariae concentrations were always high enough to sustain life cycle, and the probability of disease transmission to humans must be considered all year around. It should mentioned that dams have much higher probability of finding infected fish than in ponds, lakes and rivers (Pinlaor et al., 2013). Our results fit with results from Huay Luang dam. The infection rate varies according to fish species as well as habitats and this explains the differences in the infection prevalences and intensities observed (Pinlaor et al., 2013). Our results demonstrate the association between the intensity of metacercariae in fish and prevalence of flukes in humans, which can vary from one province to another. Similar features were observed also by Pinloar et al. (2013). Study from another Thailand area found that a reduction in the prevalence of $O$. viverrini in humans to undetectable levels reduced also the prevalence in fish to $1 \%$ (Sripa et al., 2015; personal communication).

The high, statistically significant correlation between dog, cat and human 0 . viverrini infection is of particular interest. Previous survey conducted in Thailand (Aunpromma et al., 2012) showed that infected cats $(35.51 \%)$ represent the main reservoir in an endemic areas and dogs were well behind (prevalence $=0.37 \%$ ). Earlier studies showed similar results regarding infected cats and dogs: $22.6 \%$ and $1.9 \%$ respectively (Impand et al., 1983) and $36.4 \%$ and $3.9 \%$ respectively (Enes et al., 2010). Despite dogs usually ignoring fish scraps (Aunpromma et al., 2012), dogs are apparently well accustomed to fish eating in the areas we have studied (Fig. 3). Our results show that the infected dogs (17.3\%) should also be taken into consideration as a significant reservoir. The zoonotic role of dogs should not be underestimated and should be considered in disease control strategies under similar circumstances. As stated by Sripa et al. (2015), all aspects of the life cycle/transmission process should be properly determined in regarding the contribution of other fish eating vertebrates, but may not have been adequately determined in human-wetland complexes. Moreover, despite parasite load being lower and according to our results dogs can be good indicators for the presence of the Asiatic liver fluke in endemic areas with similar characteristics to that which we recently studied.

Our results allow us to demonstrate that the ecology of transmission between vertebrates (reservoirs as well as the intermediate hosts) is based on direct associations between the elements involved in the parasite life cycle. Consequently, measures to control opisthorchiasis should consider into account various environmental components. As regards to the administration of antihelminthics to humans the treatment is only effective in the short term. It is due to parasites survival in the environment as well as in the other hosts. The other prevention strategies and control programs should be considered to break the epidemiological cycle. Analysis of the risk factors in the villages including the people education is critical element to control and eradicate opisthorchiasis (Petney et al., 2013; Sripa et al., 2015). In conclusion, an integrative approach to 0 . viverrini infection is essential to achieve the best results on the elimination or reduction of this parasite among the population living in endemic areas.

\section{Acknowledgements}

This study was supported by the Research and Development Institute Udonthani Rajabhat University. We also thank Brian Usher from the Faculty of Science of Udon Thani Rajabhat University for English editing of the manuscript.

Conflict of interest statement: We declare that we have no conflict of interest.

\section{References}

Andrews, R.H., Sithithaworn, P., Petney, T.N. (2008): Opisthorchis viverrini: an underestimated parasite in world health. Trends Parasitol., 24(11): 497 - 501. DOI: 10.1016/j.pt.2008.08.011

Aunpromma, S., Tangkanattana, P., Papirom, P., Kanjampa, P., TeSANA, S., SRIPA, B., Tangkawattana, S. (2012): High prevalence of Opisthorchis viverrini infection in reservoir hosts in four districts of Khon Kaen Province, an opisthorchiasis endemic area of Thailand. Parasitol. Int., 61(1): 60 - 64. DOI: 10.1016/j.parint.2011.08.004 CDC (2012) Opisthorchis - Resources for Health Professionals at: https://www.cdc.gov/parasites/opisthorchis/health_professionals/ index.html accesed 4.17.

Chaiputcha, K., Promthet, S., Bradshaw, P. (2015): Prevalence and risk factors for infection by Opisthorchis viverrini in an urban area of Mahasarakham Province, northeast Thailand. Asian Pac. J. Cancer Prev., 16(10): 4173 - 4176

Donthaisong, C., Arunsan, P., Sumannatral, K., Prasopdee, S., Kulsantiwong, J., Wongmaneeprateep, S., Suwannatral, A., Tesana, S. (2014): Experimental infection of Opisthorchis viverrini cercariae to the cyprinid fish, Barbonymus gonionotus. Acta Trop., 136: 118 - 122. DOI: 10.1016/j.actatropica.2014.04.022

Enes, J.E., Wages, A.J., Malone, J.B., Tesana, S. (2010): Prevalence of Opisthorchis viverrini infection in the canine and feline hosts in three villages, Khon Kaen Province, northeastern Thailand. Southeast Asian J. Trop. Med. Public Health, 41(1):, 36 - 42 Grundy-Warr, C., Andrews, R.H., Sithithaworn, P., Petney, T.N., Sripa, B., Laithavewat, L., Ziegler, A.D. (2012): Raw attitudes, wetland cultures, life-cycles: socio-cultural dynamics relating to Opisthorchis viverrini in the Mekong Basin. Parasitol. Int., 61(1): 65 - 70. DOI: 10.1016/j.parint.2011.06.015

HARINASUTA, C., HARINASUTA, T. (1984): Opisthorchis viverrini: life cycle, intermediate hosts, transmission to man and geographical distribution in Thailand. Arzneimittelforschung., 34(9B): 1164 - 1167 ImPAND, P., ThiRAChANTRA, S., Bunnag, T. (1983): Helminth faunas of rats and domestic animals and their zoonotic potential role in north and northeast Thailand. J. Parasiological Trop. Med. Assoc. Thail., 6: $105-116$

Kaewpitoon, N., Kootanavanichpong, N., Kompor, P., Chavenkun, W., Kujapun, J., Norkaew, J., Ponphimal, S., Matrakool, L., Tongtamee, 
T., Panpimanmas, S., Rujirakul, R., Padchasuwan, N., Pholsripradit, P., Eksanti, T., Phatisena, T., Loyd, R.A., Kaewpitoon, S.J. (2015): Review and current status of Opisthorchis viverrini infection at the community level in Thailand. Asian Pac. J. Cancer Prev., 16(16): $6825-6830$

Khuntikeo, N., Sithithaworn, P., Lollom, W. Namwat, N., Yongvanit, P., Thinkhamrop, B., Kiatsopit, N., Andrews, R.H., Petney, T.N. (2016): Changing patterns of prevalence in Opisthorchis viverrini sensu lato infection in children and adolescents in northeast Thailand. Acta Trop., 164: 469 - 472. DOI: 10.1016/j.actatropica.2016.10.017

Madsen, H., Dung, B.T., The, D.T. Viet, N.K., Dalsgaard, A., Van, P.T. (2015): The role of rice fields, fish ponds and water canals for transmission of fish-borne zoonotic trematodes in aquaculture ponds in Nam Dinh Province, Vietnam. Parasit. Vectors, 8(1): 625. DOI: 10.1186/s13071-015-1237-z

Miyamoto, K., Kirinoki, M., Matsuda, H., Hayashi, N., Chigusa, Y., Sinuon M., Chuor, C.M., Kitikoon, V. (2014): Field survey focused on Opisthorchis viverrini infection in five provinces of Cambodia. Parasitol. Int., 63(2): 366 - 373. DOI: 10.1016/j.parint.2013.12.003 Onsurathum, S., Pinlaor, P., Charoensuk, L., Haonon, O., Chaidee, A., Intuyod, K., Laummaunwal P., Boonmars, T., Kaewkes, W., Pinlaor, S. (2016): Contamination of Opisthorchis viverrini and Haplorchis taichui metacercariae in fermented fish products in northeastern Thailand markets. Food Control, 59: 493 - 498. DOI: 10.1016/j. foodcont.2015.06.020

Petney, T.N., Andrews, R.H., Saijuntha, W., Wenz-Mücke, A., SithiTHAWORN, P. (2013): The zoonotic, fish-borne liver flukes Clonorchis sinensis, Opisthorchis felineus and Opisthorchis viverrini. Int. J. Parasitol., 43: 1031 - 1046 DOI: 10.1016/j.jpara.2013.07.007 Pinlaor, S., Onsurathum, S., Boonmars, T., Pinlaor, P., Hongsrichan, N., Chaidee, A., Haonon, O., Limviroj, W., Tesana, S., SithiTHAWORN, P. (2013): Distribution and abundance of Opisthorchis viverrini metacercariae in cyprinid fish in Northeastern Thailand. Korean J. Parasitol., 51(6): 703 - 710. DOI: 2013.51.6.703

Prasongwatana, J., Laummaunwal, P., Boonmars, T., Pinlaor, S. (2013): Viable metacercariae of Opisthorchis viverrini in northeastern thai cyprinid fish dishes-as part of a rational program for control of 0 . viverrini-associated cholangiocarcinoma. Parasitol. Res., 112(3): 1323 - 1327. DOI: 10.1007/s00436-012-3154-9
Preuksaraj, S., Jeeradit, C., Satilthal, A., Sidofrusmi, T., KiJwannee, S., (1982): Prevalence and intensity of intestinal helminthiasis in rural Thailand 1980-1981. Con. Dis. J. 8: 221-269 (in thai).

Pungpark, S., Bunnag, D., Harinasuta, T. (1984): Albendazole in the treatment of opisthorchiasis and concomitant intestinal helminthic infections. Southeast Asian J. Trop. Med. Public Health, 15(1): 44 $-50$

Saengsamang, P., Promthet, S., Bradshaw, P. (2016): Reinfection by Opisthorchis viverrini after treatment with praziquantel. Asian Pac. J. Cancer Prev., 17(2): 857 - 862

Sithithaworn, P., Andrews, R.H., Nguyen, V., Wongsaroj, T., Sinuon, M., Odermatt, P., Nawa, Y., Liang, S., Brindley, P.J., SRipa, B. (2012): The current status of opisthorchiasis and clonorchiasis in the Mekong Basin. Parasitol. Int., 61(1): 10 - 16. DOI: 10.1016/j. parint.2011.08.014

Sithithaworn, P., Haswell-Elkins, M. (2003): Epidemiology of Opisthorchis viverrini. Acta Trop., 88(3): 187 - 194

Sithithaworn, P., Pipitgool, V., SRIsawangwong, T. Elkins, D.B., HasWELL-ELKINS, M.R. (1997): Seasonal variation of Opisthorchis viverrini infection in cyprinoid fish in north-east Thailand: implications for parasite control and food safety. Bull. World Health Organ., 75(2): 125 - 131

Sripa, B., KaewKes, S., Intapan, P.M., Maleewong, W., Brindley, P.J. (2010): Food-borne trematodiases in Southeast Asia epidemiology, pathology, clinical manifestation and control. Adv. Parasitol., 72: 305 - 350. DOI: 10.1016/S0065-308X(10)72011-X

Sripa, B., Kaewkes, S., Sithithaworn, P., Mairiang, E., Laha, T., Smout, M., Pairojkul, C., Bhudhisawasdi, V., Tesana, S., Thinkamrop. B., Bethony, J.M., LoukAS, A., BRIndLey, P.J. (2007): Liver fluke induces cholangiocarcinoma. PLoS Med., 4(7): E201. DOI: 10.1371/ journal.pmed.0040201

Sripa, B., Tangkawattana, S., Laha, T., Kaewkes, S., Mallory, F.F., SMITH, J.F., WILCOX, B.A. (2015): Toward integrated opisthorchiasis control in northeast Thailand: the Lawa project. Acta Trop., 141(Pt B): 361 - 367. DOI: 10.1016/j.actatropica.2014.07.017

Thamavit, W., Pairojkul, C., Tiwawech, D. Itoh, M., Shiral, T., Ito, N. (1993): Promotion of cholangiocarcinogenesis in the hamster liver by bile duct ligation after dimethylnitrosamine initiation. Carcinogenesis, 14(11): $2415-2417$ 